\title{
¿CUÁN PREPARADOS ESTÁN LOS JEFES TÉCNICOS PARA EJERCER UNLIDERAZGO EFECTIVO? ESTUDIO DE OPINIÓN A PARTIR DEL MARCO PARA LA BUENA DIRECCIÓN Y EL LIDERAZGO ESCOLAR ${ }^{1}$
}

\section{Teresa Yáñez $z^{2}$, Rosario Rivero ${ }^{3}$}

\begin{abstract}
RESUMEN
La literatura ha situado al liderazgo escolar como el segundo factor de la escuela más relevante para el aprendizaje de los alumnos. Debido a su importancia, las políticas nacionales y la investigación han prestado creciente atención a los directores, avanzando en la creación del Marco para la Buena Dirección y el Liderazgo Escolar (MDBLE). Sin embargo, es escasa la investigación nacional sobre el liderazgo de otros miembros del equipo directivo, lo que la literatura ha denominado como "liderazgos medios", en los que, en el caso chileno, el cargo del jefe técnico ocupa un lugar clave como líder escolar. En este contexto, este artículo evalúa la preparación de los jefes técnicos para ejercer un liderazgo efectivo derivado del MDBLE, a partir de una encuesta a 300 jefes de unidades técnico-pedagógicas. Los resultados evidencian que menos del 50\% de los jefes técnicos conoce bien el marco. Además, se perciben mediana y heterogéneamente preparados para ejercerlo, encontrándose altamente preparados para algunos componentes y dimensiones del marco, y solo un $10 \%$ afirma estar preparado, tanto para las prácticas como para los conocimientos especificados en el marco. Emergen desafíos para la mejora del liderazgo escolar, mediante un fortalecimiento de las competencias instaladas en espacios de programas de formación, como también de una mejor articulación entre las políticas educativas y el marco.
\end{abstract}

Conceptos clave: jefes técnicos, liderazgo medio efectivo, políticas educativas, estudio de opinión.

1 La presente publicación se realizó al alero de los proyectos de investigación del Centro de Desarrollo de Liderazgo Educativo (CEDLE), que corresponde a una alianza entre universidades financiada por el Ministerio de Educación de Chile. El artículo fue realizado en el marco del proyecto "Estudio de opinión a líderes escolares sobre políticas de liderazgo escolar y educacional para la retroalimentación al diseño, implementación y creación de políticas educativas", con resultados de la encuesta a jefes técnicos de 2017.

2 Investigadora independiente, Santiago, Chile. Contacto: mteresayanez@gmail.com

3 Universidad Diego Portales, Santiago, Chile. Contacto: rosario.rivero@udp.cl 


\title{
HOW PREPARED ARE INSTRUCTIONAL LEADERS TO IMPLEMENT EFFECTIVE LEADERSHIP? AN OPINION STUDY, CONCERNING THEIR PERSPECTIVES OF THE NATIONAL FRAMEWORK FOR SCHOOL LEADERSHIP
}

\begin{abstract}
International evidence situates school leadership as the second most important factor for student learning. Educational national policies and research have given increased attention to school principals, evidenced by the creation of a national framework for good governance and school leadership, known in Spanish as the Marco para la Buena Dirección y el Liderazgo Escolar (MDBLE). However, there is a dearth of national research about school leadership from the perspective of the instructional coaches. In this context, this article evaluates the degree of preparation of these middle leaders to exercise an effective leadership derived from the MDBLE, based on a survey of 300 instructional coaches. The results reveal that less than $50 \%$ of these middle leaders have a solid understanding of the framework. Furthermore, while overall they consider themselves moderately prepared, there is heterogeneity in their perceptions of how well prepared they are to implement the framework, while most consider themselves highly prepared for some components and thematic dimensions, only $10 \%$ perceive themselves to be highly prepared for all components and dimensions of the framework. This evidence points out challenges for the improvement of school leadership through a strengthening of principal competences and better articulation between educational policies and the framework.
\end{abstract}

Key concepts: effective school leadership, middle leaders, educational policies, opinion study 


\section{Introducción}

La literatura sobre calidad educativa reconoce al liderazgo escolar como el segundo factor intraescuela de mayor relevancia para mejorar el logro de aprendizajes de los estudiantes, después del impacto directo del profesor dentro del aula (Leithwood, Day, Sammons, Harris \& Hopkins, 2006; Barber \& Mourshed, 2007; Bellei, Valenzuela, Vanni y Contreras, 2014). Los estudios muestran que, en general, esta variable representa un cuarto del efecto de los establecimientos educacionales en los aprendizajes, una vez controladas las características socioculturales de los estudiantes (Hallinger \& Heck, 1998; Marzano, Waters \& McNulty, 2005). Adicionalmente, algunos estudios relacionan el liderazgo escolar con otro tipo de logro de los alumnos, por ejemplo, su motivación y su compromiso con el aprendizaje, y los indicadores de desarrollo personal y social (Agencia de Calidad de la Educación, 2015; Silins \& Mulford, 2002; Robinson, Lloyd \& Rowe, 2008).

Por su parte, tanto en el país como en el extranjero, las políticas educativas centradas en fortalecer el liderazgo escolar han proliferado de acuerdo con la evidencia y recomendaciones internacionales (Barber \& Mourshed, 2007; Pont, Nusche \& Moorman, 2009). Durante los últimos años, Chile ha avanzado en contar con un Marco para la Buena Dirección y el Liderazgo Escolar (MBDLE, en adelante), que reconoce este rasgo en los responsables escolares, independiente de la función directiva específica que desempeñen, y que no se centra exclusivamente en el director y en políticas relativas a la selección, remuneración, evaluación y formación de directores, entre otras.

En particular, el MBDLE tuvo una primera versión en 2005 — actualizada en 2015- y constituye un referente para el desarrollo del liderazgo escolar. Se organiza en cinco dimensiones claves para ejercer un liderazgo efectivo: i) construir e implementar una visión estratégica; ii) desarrollar capacidades profesionales; iii) liderar procesos de enseñanza y aprendizaje; iv) gestionar la convivencia y la participación de la comunidad escolar, y v) desarrollar y gestionar el establecimiento escolar. El MBDLE pretende articular las políticas de liderazgo y orientar a los equipos directivos acerca de conocimientos 
y prácticas para el desarrollo de su labor. Adicionalmente, entrega lineamientos para el proceso de desarrollo y formación especializada de quienes ejercen cargos directivos en los establecimientos.

Desde la evidencia nacional, variadas investigaciones estudian el liderazgo escolar desde el rol del director, enfocándose en la efectividad de sus resultados, e identifican prácticas que marcan diferencias, como son establecer metas y coordinar el trabajo pedagógico (Volante, 2012; Marfán, González, Muñoz, Pascual y Weinstein, 2012; Valenzuela y Horn, 2012). Sin embargo, más allá de estos aportes, es escasa la investigación nacional sobre el liderazgo de otros miembros del equipo directivo de los establecimientos, lo que la literatura ha denominado "liderazgos medios", en los que el cargo del jefe o encargado técnico, como se denomina en el sector público al jefe de la unidad técnico-pedagógica (conocido en los establecimientos como "jefe de UTP"), ocupa un lugar clave como líder escolar en el caso chileno.

De esta manera, en la actualidad, no se conocen estudios que evalúen el liderazgo medio de los jefes de UTP y, en particular, que se focalicen en determinar cuán preparados están los jefes técnicos para ejercer un liderazgo efectivo. En otras palabras, se ignora hoy si los jefes de UTP presentan las condiciones necesarias para ejercer de forma satisfactoria su rol, de acuerdo con las definiciones del MBDLE, tal como se define en el único instrumento de política educativa que enmarca las prácticas, conocimientos y habilidades que deberían dominar para ejercer un liderazgo efectivo.

Dada la relevancia del liderazgo escolar en el aprendizaje de los estudiantes y la falta de evidencia empírica acerca del liderazgo medio, y en particular respecto del grado de preparación de los encargados técnicos para ejercer un liderazgo efectivo de acuerdo a las definiciones del MBDLE, a partir de una encuesta presencial a 300 jefes técnicos el presente estudio aborda los siguientes objetivos: i) evaluar el conocimiento y el grado de preparación para ejercer de forma efectiva las prácticas, conocimientos y habilidades especificadas en el MBDLE, a partir de la percepción de los propios encuestados; ii) comparar si estas percepciones varían según las características 
individuales, formación y experiencia, o de los establecimientos donde trabajan los jefes técnicos, como son la dependencia y resultados en pruebas nacionales, y iii) identificar desafíos de formación que emergen para que los jefes técnicos puedan fortalecer un liderazgo escolar efectivo.

Esta información es relevante para el desarrollo de políticas que buscan reforzar el liderazgo efectivo del equipo directivo en su conjunto, mediante su formación inicial y continua, y para la implementación y articulación de otras políticas que apunten a acciones concretas, relacionadas con el ámbito pedagógico, que es central en el quehacer escolar, para mejorar el liderazgo de este importante actor del equipo directivo.

\section{Marco de referencia}

\section{Liderazgo efectivo}

La literatura sobre la efectividad del liderazgo escolar es extensa y variada, especialmente aquella que estudia a los directores de establecimientos y que identifica un conjunto de prácticasasociadas con este liderazgo (Leithwood, Day, Sammons, Harris \& Hopkins, 2006; Robinson, Lloyd \& Rowe, 2008). Sin embargo, estudios que aborden el liderazgo de otros actores del equipo directivo, vinculados a un liderazgo medio en el establecimiento, como son los jefes técnicos, han sido más bien escasos en la literatura nacional e internacional.

Desde principios de 2000 ha cambiado la conceptualización sobre liderazgo medio, desde una concepción más abocada a lo administrativo a una visión más compleja y estratégica sobre la importancia de su rol en las escuelas, con los docentes y para los estudiantes. Así, ha crecido el interés por estudiar los distintos tipos de liderazgos y, en particular, el liderazgo medio. Sin embargo, aún no hay evidencia clara sobre su rol, pues los estudios se han enfocado principalmente en las funciones y prácticas de los directores de los establecimientos educacionales, sin reflexionar acerca de la influencia de otros actores abocados a la gestión (De Nobile, 2018). 
86 ¿CUÁN PREPARADOS ESTÁN LOS JEFES TÉCNICOS PARA EJERCER UN LIDERAZGO EFECTIVO? ESTUDIO DE OPINIÓN A PARTIR DEL MARCO PARA LA BUENA DIRECCIÓN Y EL LIDERAZGO ESCOLAR - T. Yáñez, R. Rivero

Sobre la base de la reciente y vasta revisión realizada por De Nobile (2018), es posible acercarnos a la definición y concreción de los roles de los líderes medios. La literatura más reciente los define como aquellos que, a partir de sus prácticas, conducen a la creación de equipos y a la mejora en la calidad del trabajo; y que, además, están vinculados con los procesos de enseñanza-aprendizaje en el aula.

Por su parte, Cortez y Zoro (2016) realizan una revisión de la literatura nacional e internacional sobre el tema, y definen a quienes ejercen el liderazgo medio como aquellos que "trabajan cotidianamente con docentes de aula y estudiantes, apoyando y proporcionando liderazgo pedagógico y formativo, además de realizar varias funciones de gestión" (p. 6).

Aun cuando la conceptualización sobre los liderazgos medios no es única, existe una concepción común que apunta a entender lo académico y lo no académico en las escuelas como áreas interdependientes, apuntando la literatura más reciente a fomentar el trabajo colaborativo entre ambas.

Respecto de la formación de los líderes medios, desde la revisión de un programa de formación australiano, Flückiger, Lovett, Dempster y Brown (2015) proponen que esta formación debe enfocarse en entregar una formación profesional a este actor clave de los equipos directivos, que vincule las habilidades de liderazgo pedagógico con la implementación de prácticas concretas al interior de los establecimientos escolares. Habilidades destacadas en la formación se refieren al proceso de retroalimentación permanente a los docentes, promoción de prácticas colaborativas entre pares y entrega de dirección estratégica y desarrollo de las asignaturas, entre otras.

\section{¿Qué sabemos sobre el liderazgo de los jefes técnicos?}

En el caso del liderazgo medio en Chile y su conceptualización, existen distintos matices y funciones, los que se reúnen en el cargo interno de "jefe de la unidad técnico- pedagógica". Carbone (2008), a partir de un estudio empírico y teórico encargado por el MINEDUC, en el marco del MBDLE, genera una definición de este rol en Chile, con foco en su liderazgo para los procesos del área pedagógica- curricular: 
Es el responsable técnico de la dimensión pedagógica-curricular, liderando procesos de desarrollo de las actividades realizadas en ese ámbito. Entre las prácticas que competen a este cargo se encuentra: asegurar la existencia de información útil para la toma de decisiones, gestionar los recursos con que cuenta el establecimiento (materiales y recursos humanos), supervisar y acompañar el trabajo de los docentes, asegurar la implementación de metodologías y prácticas pedagógicas en el aula, realizar seguimiento de los procesos curriculares. (Carbone, 2008, p. 17)

El caso chileno cuenta con escasos estudios sobre el rol de los liderazgos medios al interior de los establecimientos. A pesar de la relevancia de sus cargos en el contexto escolar y de tener funciones reconocidas por el MINEDUC, los jefes técnicos han sido escasamente investigados y no han contado con formación especializada para su desempeño.

De acuerdo con la relevancia del liderazgo, el rol de los jefes técnicos y el aumento de iniciativas orientadas a fortalecer las capacidades de los equipos escolares para un liderazgo efectivo, la investigación en Chile ha avanzado en al menos dos áreas: i) estudios que buscan caracterizar a los equipos directivos en cuanto a su formación, prácticas principales y su influencia, y ii) estudios de caso y revisiones para identificar desafíos formativos y para el ejercicio del cargo.

En relación con el primer grupo, MINEDUC (2016) publicó un estudio que caracterizaba a los equipos directivos de los establecimientos educacionales subvencionados en Chile, realizando encuestas presenciales a 350 directores y 250 jefes técnicos de contextos urbanos. Se observó que el $77 \%$ de jefes técnicos tiene formación de posgrado y que, entre estos, $47 \%$ consta de diplomado, $51 \%$ de magíster y $1 \%$ de doctorado. En cuanto a las temáticas de las especializaciones, tanto los diplomados como los magísteres se relacionan con el liderazgo y gestión escolar, el currículo y la evaluación, con un bajo porcentaje especializado en temas de inclusión y equidad, desarrollo profesional, gestión de proyectos, prácticas de enseñanza y aprendizaje, entre otros. Consistentemente, 
se observó que las prácticas más realizadas por los jefes técnicos se relacionan con el área de gestión del currículo y de contingencias (resolver problemas particulares de estudiantes), con una menor frecuencia de prácticas de conducción y de desarrollo profesional. En general, los principales hallazgos apuntan a que los directores cuentan con mayor experiencia en liderazgo que los jefes técnicos, tanto en experiencia previa como en formación especializada. Las áreas más débiles en el desarrollo de la gestión se relacionan con la formación y participación, el desarrollo profesional y la gestión de la comunidad interna. El estudio concluye que existe una importante necesidad de mejora relacionada con la formación y capacitación de los profesionales, y que responda a sus necesidades.

Rodríguez (2017), por su parte, analizó la influencia de los procesos de gestión curricular de los jefes técnicos en las prácticas pedagógicas de docentes en establecimientos municipales de las regiones del Biobío y Araucanía. Para ello, realizó un estudio con enfoque mixto, a partir de la aplicación de encuestas a 576 jefes técnicos y entrevistas y observaciones en cinco establecimientos educacionales de la región del Biobío. Los principales hallazgos indican que, si bien existe un alto porcentaje de jefes técnicos que afirman haber recibido formación en relación con su cargo (95\%), la especialización se basa principalmente en actividades de desarrollo profesional. En cuanto al conocimiento de los jefes técnicos sobre distintos elementos normativos y pedagógicos, se observa que las áreas en que hay mayor carencia son la teoría del aprendizaje, la didáctica general y la didáctica de las disciplinas. Además, el estudio revela la influencia del jefe técnico en el contexto de preparación de la enseñanza, principalmente en la planificación y organización curricular con los docentes, lo que se posiciona como un ámbito necesario en términos de preparación y formación.

En el segundo grupo se encuentran estudios de casos y revisiones de literatura. Quiroga y Aravena (2017) identificaron y caracterizaron los desafíos de los jefes técnicos noveles en suprimer año de ejercicio, a partir de un estudio de casos con diversas etapas de consultas. Entre los principales hallazgos, el estudio indica que, si bien los jefes técnicos aseguran haber realizado cursos de especialización 
una vez en el cargo, reconocen carecer de conocimientos técnicos y estrategias para la resolución de conflictos y el mejoramiento de las prácticas pedagógicas. Los autores aseguran que esto se debe a que hay una carencia en Chile de programas de formación dirigidos específicamente al cargo de jefe técnico, lo que Flückiger et al. (2015) también destacan como una necesidad desde la perspectiva internacional.

Desde una revisión de literatura sobre el caso chileno (Cortez y Zoro, 2016), se identifican los principales requerimientos formativos en Chile para el cargo de liderazgo de los jefes técnicos, apuntando a "potenciar la construcción de un liderazgo que aborde de manera interdependiente lo pedagógico y lo formativo, y que avance hacia un liderazgo colaborativo posibilitando contar con equipos de trabajo requeridos para la innovación y mejora de nuestras escuelas y liceos" (p. 9). Estas necesidades se refieren a la implementación de instancias de aprendizaje formal sobre el liderazgo, al desarrollo de innovaciones para resolver problemas colectivamente y a la formación de un liderazgo pedagógico, entendido como la capacidad de aprendizaje colectivo que fomente la mejora de las prácticas de enseñanza y aprendizaje, y de espacios de desarrollo del liderazgo formativo.

Ulloa y Gajardo (2017), a partir del proceso de formación de jefes técnicos en la región del Biobío, identifican como elementos deficitarios de las prácticas de los jefes técnicos el monitoreo curricular que realizan sobre el progreso de los aprendizajes de los estudiantes y sus habilidades de liderazgo y gestión del cambio.

Por último, en un estudio de caso que analiza las dificultades de los jefes técnicos para ejercer su rol en liceos de la región de la Araucanía, Beltrán (2014) destaca, desde el ámbito formativo, la necesidad de contar con competencias de coordinación de actividades pedagógicas y de comunicación con el cuerpo docente, que permitan mejorar el desarrollo 
90 ¿CUÁN PREPARADOS ESTÁN LOS JEFES TÉCNICOS PARA EJERCER UN LIDERAZGO EFECTIVO? ESTUDIO DE OPINIÓN A PARTIR DEL MARCO PARA LA BUENA DIRECCIÓN Y EL LIDERAZGO ESCOLAR - T. Yáñez, R. Rivero

de sus funciones y el resultado dentro del proceso de aprendizaje de los estudiantes.

Marco para la Buena Dirección de Liderazgo Escolar (MBDLE)

De acuerdo con la política educativa chilena, la definición de un liderazgo escolar efectivo ha sido recogida por el MBDLE, propuesto por el Ministerio de Educación en 2005 y actualizado en 2015. Este marco es una guía orientadora para el desarrollo del liderazgo de los equipos directivos de los establecimientos y no presenta un carácter prescriptivo. El objetivo principal del documento es ser un referente sobre las prácticas y conocimientos para el desarrollo del liderazgo en el país, orientar la acción del liderazgo escolar efectivo y servir de guía para los procesos de formación especializada (MINEDUC, 2015).

El MBDLE describe prácticas, recursos personales y conocimientos fundamentales para el desarrollo del liderazgo. Incluye una descripción, en cinco dimensiones, del quehacer de los líderes escolares que conforman los equipos directivos, incluyendo así la labor del jefe técnico-pedagógico en los establecimientos. Las prácticas describen el hacer en términos generales, para gestionar los establecimientos educativos de manera efectiva. Las cinco dimensiones que agrupan las prácticas son: i) construyendo e implementando una visión estratégica compartida; ii) desarrollando las capacidades profesionales; iii) liderando y monitoreando los procesos de enseñanza y aprendizaje; iv) gestionando la convivencia y la participación de la comunidad escolar, y v) desarrollando y gestionando la organización del establecimiento escolar. A su vez, el documento señala que, para dar cumplimiento a estas prácticas, se requieren recursos personales organizados en tres ámbitos: i) principios, ii) habilidades y iii) conocimientos profesionales.

\section{Metodología}

\section{Dimensiones de análisis}

Para estudiar el grado de preparación autopercibido por los jefes técnicos, se identificaron diversas prácticas y conocimientos asociadas a las dimensiones del MBDLE, pertinentes al quehacer específico 
de los jefes técnicos. Con base en las funciones principales de los jefes técnicos en los establecimientos, estas dimensiones se centran mayoritariamente en construir e implementar una visión estratégica compartida, desarrollar las capacidades profesionales y liderar y monitorear los procesos de enseñanza y aprendizaje. La tabla 1 muestra las áreas de preparación y los aspectos evaluados para cada una de ellas, a partir de una priorización de lo descrito en el marco y de acuerdo con las áreas de trabajo de los jefes técnicos.

Tabla 1.

Componentes de preparación y aspectos evaluados.

\begin{tabular}{ll}
\hline $\begin{array}{c}\text { Componentes de } \\
\text { preparación }\end{array}$ & \multicolumn{1}{c}{ Aspectos evaluados } \\
\hline & Concepciones teóricas contemporáneas acerca del liderazgo. \\
& Conocimientos acerca de inclusión y equidad y estrategias para \\
& promoverlas. \\
& Conocimiento de las políticas nacionales de educación, \\
& normativa nacional y local. \\
& Conocimiento en mejoramiento y cambio escolar. \\
& Conocimiento acerca de bases curriculares, planes y programas \\
& de estudio, y estándares de aprendizaje. \\
& Conocimiento respecto de evaluación a nivel institucional \\
& y de aula, sus instrumentos, metodologías de análisis y \\
& sistematización de información. \\
& Conocimiento acerca de evaluaciones de aprendizaje y \\
& aplicaciones adecuadas. \\
& Conocimiento acerca de metodologías y prácticas efectivas de \\
& enseñanza-aprendizaje. \\
& Conocimiento respecto de observación y retroalimentación a \\
& profesores en relación con sus prácticas de aula. \\
\hline & Capacidad de promover y participar en el desarrollo profesional \\
& de los docentes. \\
& Capacidad de dirigir los procesos de enseñanza aprendizaje, \\
& planificando, coordinando y evaluando las prácticas de \\
enseñanza. & Capacidad de instaurar una cultura de altas expectativas en la \\
comunidad educativa. & Capacidad de movilizar el establecimiento hacia la mejora \\
continua. & Capacidad de elaborar planes de mejoramiento y las metas \\
institucionales y de aprendizaje asociadas. & Capacidad para participar en los procesos de selección de los \\
& docentes. \\
\hline Prácticas & \\
\hline &
\end{tabular}

Fuente: Elaboración propia a partir de MBDLE. 


\section{Recolección de información}

Mediante la aplicación de una encuesta presencial, se recolectó información acerca de la percepción de los jefes técnicos de su nivel de conocimiento del marco y de su preparación respecto de las áreas descritas. La encuesta se aplicó de manera presencial en establecimientos educacionales de la Región Metropolitana de Santiago, entre junio y agosto del 2017, con una muestra de 300 jefes técnicos. Las tablas 2 y 3 muestran la operacionalización de los indicadores evaluados en las áreas de conocimientos y prácticas, identificando la dimensión del marco a la que pertenecen.

Tabla 2.

Operacionalización área de conocimientos.

\begin{tabular}{|c|c|}
\hline Dimensión & Indicadores \\
\hline \multirow{3}{*}{$\begin{array}{l}\text { Construyendo e } \\
\text { implementando una } \\
\text { visión estratégica } \\
\text { compartida. }\end{array}$} & Mejoramiento y cambio escolar. \\
\hline & $\begin{array}{l}\text { Concepciones teóricas contemporáneas del liderazgo } \\
\text { escolar. }\end{array}$ \\
\hline & $\begin{array}{l}\text { Conocimiento de las políticas nacionales de educación, } \\
\text { normativa nacional y local. }\end{array}$ \\
\hline \multirow[t]{4}{*}{$\begin{array}{l}\text { Liderando los procesos de } \\
\text { enseñanza y aprendizaje. }\end{array}$} & $\begin{array}{l}\text { Bases curriculares, planes y programas de estudio, y } \\
\text { estándares de aprendizaje. }\end{array}$ \\
\hline & $\begin{array}{l}\text { Observación y retroalimentación a profesores con } \\
\text { relación a sus prácticas de aula. }\end{array}$ \\
\hline & $\begin{array}{l}\text { Conocimiento acerca de evaluaciones de aprendizaje y } \\
\text { aplicaciones adecuadas. }\end{array}$ \\
\hline & $\begin{array}{l}\text { Metodologías y prácticas efectivas de enseñanza- } \\
\text { aprendizaje. }\end{array}$ \\
\hline $\begin{array}{l}\text { Gestionando la convivencia } \\
\text { y la participación de la } \\
\text { comunidad escolar. }\end{array}$ & $\begin{array}{l}\text { Conocimientos sobre inclusión y equidad y estrategias } \\
\text { para promoverlas. }\end{array}$ \\
\hline $\begin{array}{l}\text { Desarrollando y } \\
\text { gestionando el } \\
\text { establecimiento escolar. }\end{array}$ & $\begin{array}{l}\text { Procesos de evaluación en los niveles institucional y } \\
\text { de aula, sus instrumentos, metodologías de análisis y } \\
\text { sistematización de información. }\end{array}$ \\
\hline
\end{tabular}

Fuente: Elaboración propia a partir de MBDLE. 
Tabla 3.

Operacionalización área de prácticas.

\begin{tabular}{ll}
\hline Dimensión & Indicadores \\
\hline $\begin{array}{l}\text { Construyendo e } \\
\text { implementando una visión } \\
\text { estratégica compartida. }\end{array}$ & $\begin{array}{l}\text { Instaurar una cultura de altas expectativas en la } \\
\text { comunidad educativa. }\end{array}$ \\
\cline { 2 - 2 } $\begin{array}{l}\text { Desarrollando las } \\
\text { capacidades profesionales. }\end{array}$ & $\begin{array}{l}\text { Elaborar los planes de mejoramiento y las metas } \\
\text { institucionales y de aprendizaje asociadas. }\end{array}$ \\
\cline { 2 - 2 } $\begin{array}{l}\text { Participar en los procesos de selección de los docentes. } \\
\text { los docentes. }\end{array}$ \\
\hline $\begin{array}{l}\text { Liderando los procesos de } \\
\text { enseñanza y aprendizaje. }\end{array}$ & $\begin{array}{l}\text { Movilizar el establecimiento hacia la mejora continua. } \\
\text { planificando, coordinando y evaluando las prácticas de } \\
\text { enseñanza. }\end{array}$ \\
\hline
\end{tabular}

Fuente: Elaboración propia a partir de MBDLE.

\section{Marco muestral y características de la muestra}

El universo del estudio corresponde a los 1.961 jefes técnicos de establecimientos de educación básica y media de la Región Metropolitana. La tabla 4 presenta la distribución por dependencia del universo de establecimientos y de la muestra del estudio, en la que se observa una proporción equivalente de la muestra con la dependencia de los establecimientos del universo ${ }^{4}$.

Tabla 4.

Características de los jefes de técnicos de la muestra según dependencia.

\begin{tabular}{lcccc}
\hline \multirow{2}{*}{ Dependencia } & \multicolumn{2}{c}{ Universo } & \multicolumn{2}{c}{ Muestra } \\
\cline { 2 - 5 } & Número & $\%$ & Número & $\%$ \\
\hline Municipal & 644 & 33 & 98 & 33 \\
\hline Particular subvencionado & 1.071 & 55 & 164 & 54 \\
\hline Particular pagado & 246 & 13 & 38 & 13 \\
\hline Total & 1.961 & 100 & 300 & 100 \\
\hline
\end{tabular}

La tabla 5 presenta las características de los jefes técnicos de la muestra y sus establecimientos. En cuanto a género, se observa una proporción de la muestra consistente con lo informado por

4 La encuesta se aplicó a una muestra probabilística y representativa de los jefes técnicos de la Región Metropolitana. El error muestral asociado es de un 5\%, considerando un nivel de confianza de $95 \%$. 
un estudio nacional de liderazgo escolar realizado por MINEDUC $(2016)^{5}$, que identificó que un 68\% de los jefes técnicos son mujeres y un 32\% son hombres (Rivero, Yáñez, Hurtado y Strello, 2017). En la muestra se observa una mayor proporción de jefes técnicos hombres en el sector particular pagado y menos mujeres al comparar entre dependencias. En términos de edad, los establecimientos particulares pagados destacan por una menor proporción de jefes técnicos menores de 40 años, al comparar con las otras dos dependencias. Respecto del nivel de formación, se obtiene que un 64\% de los jefes técnicos cuenta con el grado de magíster y que más de la mitad, un $55 \%$, tiene seis años o menos de experiencia en el cargo. Por último, la distribución por tramos de puntaje SIMCE señala que la mayoría de los establecimientos municipales se concentra en la categoría baja de resultados Simce, de 241 o menos (68\%); el sector particular subvencionado de la muestra concentra a casi la mitad de los casos en la categoría media de puntajes entre 242 a 283 (48\%) y los establecimientos particulares pagados se concentran en la categoría de mayor puntaje de 284 puntos o más (84\%).

5 Estudio nacional realizado en zonas urbanas, entrevistando directores, jefes de UTP e inspectores generales. Muestra jefes técnicos: $\mathrm{N}=250$, error muestral $=6 \%$. 
Tabla 5.

Distribución de género y edad del universo y muestra.

\begin{tabular}{|c|c|c|c|c|c|}
\hline \multicolumn{2}{|l|}{ Características } & Total & Municipal & $\begin{array}{c}\text { Particular } \\
\text { subvencionado }\end{array}$ & $\begin{array}{c}\text { Particular } \\
\text { pagado }\end{array}$ \\
\hline \multicolumn{2}{|l|}{ Número } & 300 & 98 & 164 & 38 \\
\hline \multirow{2}{*}{ Género } & Hombre & $32 \%$ & $26 \%$ & $33 \%$ & $45 \%$ \\
\hline & Mujer & $68 \%$ & $74 \%$ & $67 \%$ & $55 \%$ \\
\hline \multirow{3}{*}{ Edad } & Hasta 40 años & $31 \%$ & $28 \%$ & $35 \%$ & $21 \%$ \\
\hline & 40 a 55 años & $29 \%$ & $25 \%$ & $28 \%$ & $42 \%$ \\
\hline & 55 años o más & $40 \%$ & $47 \%$ & $37 \%$ & $37 \%$ \\
\hline \multirow{2}{*}{$\begin{array}{l}\text { Grado más alto } \\
\text { alcanzado }\end{array}$} & $\begin{array}{l}\text { Diplomado o sin } \\
\text { especialización }\end{array}$ & $36 \%$ & $31 \%$ & $42 \%$ & $24 \%$ \\
\hline & Magíster & $64 \%$ & $69 \%$ & $58 \%$ & $76 \%$ \\
\hline \multirow{3}{*}{$\begin{array}{l}\text { Experiencia del } \\
\text { jefe técnico en } \\
\text { el cargo }\end{array}$} & 6 años o menos & $55 \%$ & $58 \%$ & $52 \%$ & $55 \%$ \\
\hline & entre 7 y 14 & $30 \%$ & $30 \%$ & $29 \%$ & $30 \%$ \\
\hline & 15 o más & $16 \%$ & $18 \%$ & $16 \%$ & $16 \%$ \\
\hline \multirow{3}{*}{$\begin{array}{l}\text { Puntaje SIMCE } \\
\text { (4 básico } \\
\text { matemática } \\
2016)\end{array}$} & 241 o menos & $40 \%$ & $65 \%$ & $32 \%$ & $5 \%$ \\
\hline & 242 a 283 & $37 \%$ & $31 \%$ & $48 \%$ & $11 \%$ \\
\hline & 284 o más & $23 \%$ & $4 \%$ & $20 \%$ & $84 \%$ \\
\hline
\end{tabular}

Fuente: Elaboración propia.

\section{Análisis}

Para abordar los objetivos de este estudio y medir el grado de preparación de los jefes técnicos con relación a los conocimientos y prácticas definidas en el marco que corresponden a su trabajo, se utilizó la misma metodología seguida para el análisis del grado de preparación de los directores para ejercer un liderazgo efectivo, de acuerdo con las definiciones del MBDLE (Rivero, Yáñez y Hurtado, 2019). Se elaboraron entonces dos índices que clasifican a los jefes técnicos en baja, media o alta preparación. Para clasificar a los jefes técnicos en estas categorías, se construyeron dos puntos de corte en función de la suma de las respuestas en cada ítem. La categoría alta preparación de los índices considera a los jefes técnicos con al menos un 70\% de los ítems con respuestas de preparación alta. En la categoría media se consideran casos que tienen menos del 70\% de los ítems con respuesta de preparación alta y menos del 30\% en preparación baja. La categoría baja preparación corresponde a quienes declaran tener una preparación baja en más de un 30\% de los ítems. 
Por último, para estudiar si existen diferencias en el grado de preparación entre jefes técnicos, se estudió la distribución de las categorías de estos índices según características personales (experiencia y formación) y de sus establecimientos (dependencia administrativa y resultados SIMCE). Con el fin de establecer la existencia de diferencias significativas entre grupos, se usó el estadístico chi-cuadrado, considerando significativas aquellas diferencias registradas en un nivel de 95\% de confianza.

\section{Resultados}

\section{Cocimiento del MBDLE}

En términos generales, los resultados muestran que los jefes técnicos declaran que conocen el MBDLE: un 49\% que afirma conocerlo muy bien, un $44 \%$ conocerlo algo y un 7\% no conocerlo. Los jefes técnicos de establecimientos municipales son los que declaran conocerlo en mayor medida respecto de los encuestados de establecimientos de otras dependencias administrativas, siendo los jefes técnicos de establecimientos particulares pagados quienes lo conocen en menor medida.

Tabla 6.

Nivel de conocimiento del MBDLE.

\begin{tabular}{lcccc}
\hline & Total & Municipal & $\begin{array}{c}\text { Particular } \\
\text { subvencionado }\end{array}$ & $\begin{array}{c}\text { Particular } \\
\text { pagado }\end{array}$ \\
\hline Sí, lo conozco muy bien & $49 \%$ & $64 \% *$ & $43 \%$ & $37 \%$ \\
\hline Lo conozco algo & $44 \%$ & $34 \%$ & $50 \%^{\mathrm{a}}$ & $42 \%$ \\
\hline $\begin{array}{l}\text { No lo conozco para } \\
\text { nada }\end{array}$ & $7 \%$ & $2 \%$ & $7 \%$ & $21 \% *$ \\
\hline Total & $100 \%$ & $100 \%$ & $100 \%$ & $100 \%$ \\
\hline
\end{tabular}

Total de jefes técnicos $\mathrm{N}=300$.

* Las diferencias entre los porcentajes correspondientes a cada dependencia administrativa, respecto de las otras dos dependencias, son estadísticamente significativas con un 95\% de confianza. (a) Las diferencias entre los porcentajes correspondientes a los establecimientos municipales y particulares subvencionados son estadísticamente significativas con un $95 \%$ de confianza. 
Grado de preparación para ejercer un liderazgo efectivo

A continuación se analiza el grado de preparación respecto de prácticas y conocimientos del marco, y de las dimensiones que atañen de manera más directa al trabajo del jefe técnico, identificando las posibles diferencias según las características de los jefes técnicos y su establecimiento. En segundo lugar se realiza un análisis comparativo del grado de preparación entre las distintas áreas y dimensiones.

\section{Componente "prácticas del MBDLE"}

Los datos muestran que los jefes técnicos se perciben medianamente preparados para ejercer ciertas prácticas efectivas definidas en el marco. En particular, un 19\% de los jefes técnicos queda clasificado en la categoría altamente preparado del índice de prácticas, un $60 \%$ en la categoría preparación media y $21 \%$ en la baja.

$\mathrm{Al}$ analizar los resultados del índice según características de los jefes técnicos y sus establecimientos, se observa diferencias significativas de acuerdo con el nivel de estudios, los años de experiencia en el cargo y la dependencia administrativa. Los jefes técnicos que tienen estudios de magíster quedan clasificados en mayor proporción en el nivel alto de índice de prácticas que aquellos que no tienen dichos estudios (25 y 14\% respectivamente), y en menor proporción en el nivel bajo ( 15 y 25\%). Quienes tienen más años de experiencia en el cargo se sienten más preparados que aquellos con menos años de experiencia, quedando en el nivel alto de preparación un 34\% de los jefes técnicos con 15 o más años de experiencia, 25\% aquellos que tienen entre 7 y 14 años de experiencia y $15 \%$ aquellos con 6 años o menos. En cuanto a la dependencia del establecimiento, los jefes técnicos de establecimientos particulares pagados quedan clasificados en mayor proporción que sus pares de otras dependencias en el nivel medio del índice, y en menor proporción en el nivel bajo de preparación. No se observan diferencias según los resultados Simce de los establecimientos.

Respecto de las prácticas específicas analizadas, la tabla 7 muestra que los jefes técnicos se perciben con mayor nivel de preparación en las prácticas asociadas a elaborar el programa de 
mejoramiento e instalar una cultura de altas expectativas —más de un tercio de los jefes técnicos se percibe como altamente preparado-, seguidas de las prácticas de participar en procesos de selección de los docentes y movilizar al establecimiento hacia la mejora continua. En las prácticas de promoción del desarrollo profesional docente y participar en los procesos de selección se perciben con menor nivel de preparación, con casi un $20 \%$ que queda clasificado en el nivel bajo de preparación.

Tabla 7.

Grado de preparación en prácticas de los jefes técnicos.

\begin{tabular}{lccc}
\hline Práctica & Alta & Media & Baja \\
\hline $\begin{array}{l}\text { a. Promover y participar en el desarrollo profesional } \\
\text { de los docentes. }\end{array}$ & $21 \%$ & $62 \%$ & $17 \%$ \\
\hline $\begin{array}{l}\text { b. Dirigir los procesos de enseñanza aprendizaje, } \\
\text { planificando, coordinando y evaluando las prácticas } \\
\text { de enseñanza. }\end{array}$ & $29 \%$ & $61 \%$ & $10 \%$ \\
\hline $\begin{array}{l}\text { c. Instaurar una cultura de altas expectativas en la } \\
\text { comunidad educativa. }\end{array}$ & $35 \%$ & $55 \%$ & $11 \%$ \\
$\begin{array}{l}\text { d. Movilizar el establecimiento hacia la mejora } \\
\text { continua. }\end{array}$ & $31 \%$ & $60 \%$ & $9 \%$ \\
$\begin{array}{l}\text { e. Elaborar planes de mejoramiento y las metas } \\
\text { institucionales y de aprendizaje asociadas. }\end{array}$ & $34 \%$ & $56 \%$ & $10 \%$ \\
\hline $\begin{array}{l}\text { f. Participar en los procesos de selección de los } \\
\text { docentes. }\end{array}$ & $32 \%$ & $51 \%$ & $17 \%$ \\
\hline
\end{tabular}

$\mathrm{Al}$ analizar los ítems de preparación, se observan diferencias significativas en algunos, según características de los jefes técnicos y de su establecimiento. Así, respecto de dirigir los procesos de enseñanza y aprendizaje, existen diferencias según los estudios alcanzados por el jefe técnico y los años de experiencia en el cargo, siendo aquellos que tienen estudios de magíster y más años de experiencia quienes se perciben mejor preparados para ejercer esta práctica.

La práctica referida a movilizar al establecimiento hacia la mejora continua presenta diferencias significativas según los años de experiencia en el cargo del jefe técnico: los jefes técnicos con más años de experiencia se sienten mejor preparados que sus pares con menos años de trabajo en el cargo; en cuanto a los resultados Simce del establecimiento, aquellos que trabajan en los con mejores 
resultados quedan clasificados en menor proporción en el nivel bajo del índice.

La práctica asociada a elaborar planes de mejoramiento y metas institucionales y de aprendizaje de los estudiantes presenta diferencias según nivel de estudios y años de experiencia del jefe técnico, así como según dependencia administrativa. Los jefes técnicos con estudios de magíster y más años de experiencia en el cargo se sienten mejor preparados para elaborar los planes, quedando en el nivel alto un $40 \%$ de los jefes que tienen magíster y $23 \%$ quienes no lo tienen, y un $43,5 \%$ de aquellos con 15 o más años de experiencia y un $42 \%$ aquellos que tienen entre 7 y 14 años, en contraste con quienes tienen 6 años o menos de experiencia en el cargo, que quedan en el nivel alto en un $27 \%$ de los casos. Las diferencias por dependencia se observan en el nivel de baja preparación: los establecimientos particulares subvencionados tienen una mayor proporción de jefes técnicos que se sienten menos preparados respecto de sus pares, y los de establecimientos particulares pagados en mayor proporción que los municipales, lo que puede relacionarse con la no obligatoriedad de generar este tipo de instrumento de política.

Respecto de participar en procesos de selección docente, se observan diferencias según dependencia administrativa, resultados Simce y años de experiencia en el cargo del jefe técnico. Los jefes técnicos de establecimientos particulares pagados y subvencionados se perciben mejor preparados que los de establecimientos municipales para realizar esta práctica, y los jefes técnicos de establecimientos con bajos resultados en las pruebas Simce se sienten menos preparados que aquellos de establecimientos con buenos resultados. Por su parte, los jefes técnicos con más años de experiencia en el cargo se perciben con un mayor nivel de preparación que aquellos con menos experiencia.

En síntesis, de acuerdo con las características de los establecimientos y de los jefes técnicos, se observan diferencias en el nivel de preparación de prácticas según años de experiencia en cuatro de seis ítems; según nivel de estudios en dos de seis ítems; según dependencia administrativa en dos de seis indicadores, y en 
dos de seis ítems por resultados Simce. En este sentido, los años de experiencia en el cargo entregarían mayores herramientas y una mejor preparación para implementar las diferentes prácticas definidas en el marco.

\section{Componente "conocimientos del MBDLE"}

Con relación a la percepción del componente de conocimientos, los resultados revelan que los jefes técnicos se perciben medianamente preparados, al igual que en el caso de las prácticas, con un 60\% de ellos en la categoría preparación media del índice, en comparación con un $17 \%$ que queda clasificado en la categoría de alta preparación y un $23 \%$ en la categoría baja. Al analizar el grado de preparación sobre conocimientos según las características de los jefes técnicos y sus establecimientos, no se encuentran diferencias significativas para el índice.

Respecto de los conocimientos específicos analizados, la tabla 8 muestra que los jefes técnicos se perciben con mayor nivel de preparación en instrumentos curriculares y observación y retroalimentación del trabajo docente, con un tercio aproximadamente que se percibe altamente preparado. Políticas nacionales, concepciones acerca del liderazgo e inclusión y equidad son aquellos en los que los jefes técnicos declaran un menor nivel de preparación, con un 25\% o más que se ubica en bajo nivel de preparación. 
Tabla 8.

Grado de preparación en conocimientos de los jefes técnicos.

\begin{tabular}{|c|c|c|c|}
\hline & Alta & Media & Baja \\
\hline $\begin{array}{l}\text { a. Concepciones teóricas contemporáneas acerca del } \\
\text { liderazgo (prácticas, tipos de liderazgo, estrategias } \\
\text { para la mejora escolar). }\end{array}$ & $21 \%$ & $55 \%$ & $25 \%$ \\
\hline $\begin{array}{l}\text { b. Conocimientos sobre inclusión y equidad y } \\
\text { estrategias para promoverlas. }\end{array}$ & $15 \%$ & $60 \%$ & $25 \%$ \\
\hline $\begin{array}{l}\text { c. Conocimiento en mejoramiento y cambio escolar } \\
\text { (autoevaluación, planificación, monitoreo, evaluación, } \\
\text { uso y análisis de datos). }\end{array}$ & $25 \%$ & $63 \%$ & $12 \%$ \\
\hline $\begin{array}{l}\text { d. Conocimiento sobre bases curriculares, planes y } \\
\text { programas de estudio, y estándares de aprendizaje. }\end{array}$ & $36 \%$ & $56 \%$ & $8 \%$ \\
\hline $\begin{array}{l}\text { e. Conocimiento sobre evaluación a nivelinstitucional } \\
\text { y de aula, sus instrumentos, metodologías de análisis } \\
\text { y sistematización de información. }\end{array}$ & $28 \%$ & $58 \%$ & $15 \%$ \\
\hline $\begin{array}{l}\text { f. Conocimiento sobre evaluaciones de aprendizaje y } \\
\text { aplicaciones adecuadas. }\end{array}$ & $28 \%$ & $61 \%$ & $11 \%$ \\
\hline $\begin{array}{l}\text { g. Conocimiento sobre metodologías y prácticas } \\
\text { efectivas de enseñanza-aprendizaje. }\end{array}$ & $27 \%$ & $60 \%$ & $13 \%$ \\
\hline $\begin{array}{l}\text { h. Conocimiento sobre observación y } \\
\text { retroalimentación a profesores con relación a sus } \\
\text { prácticas de aula. }\end{array}$ & $34 \%$ & $58 \%$ & $8 \%$ \\
\hline $\begin{array}{l}\text { i. Conocimiento de las políticas nacionales de } \\
\text { educación, normativa nacional y local (ej.: Ley } \\
\text { General de educación, Estatuto Docente, Ley SEP, Ley } \\
\text { de Calidad y Equidad). }\end{array}$ & $19 \%$ & $51 \%$ & $29 \%$ \\
\hline
\end{tabular}

Al analizar los ítems de conocimiento se observan diferencias significativas según características de los jefes técnicos y del establecimiento. Respecto del conocimiento sobre concepciones teóricas contemporáneas de liderazgo, se perciben diferencias según puntaje Simce, años de experiencia del jefe técnico y nivel de estudios. Así, los jefes técnicos con hasta 14 años de experiencia en el cargo y que se desempeñan en establecimientos que presentanun menor puntaje Simce, declaran un menor nivel de preparación en este conocimiento respecto de jefes técnicos de establecimientos con mejores resultados Simce y más años de experiencia. Adicionalmente, aquellos con estudios de magíster declaran una mayor preparación respecto de quienes no tienen dichos estudios, y a la inversa en el caso de bajo nivel de conocimiento. De esta manera, se observa que los años de experiencia del jefe técnico y los estudios de magíster marcan 
diferencias respecto de la percepción de preparación de los jefes técnicos con relación a este conocimiento, al igual que los resultados del establecimiento: a menores resultados mayor proporción de jefes técnicos que se ubica en bajo nivel de conocimiento sobre concepciones contemporáneas acerca de liderazgo.

También se observan diferencias en relación con el conocimiento sobre mejoramiento y cambio educativo, según dependencia administrativa, puntaje Simce y estudios de magíster del jefe técnico. Los jefes técnicos de establecimientos municipales declaran en menor medida bajo nivel de conocimiento sobre mejoramiento y cambio escolar que aquellos de establecimientos particulares subvencionados y pagados. Llama la atención que los jefes técnicos de establecimientos con resultados más bajos se perciben mejor preparados en este conocimiento respecto de sus pares de establecimientos con mejores resultados, y a su vez en menor proporción en el nivel bajo de preparación. Por otra parte, los que tienen estudios de magíster se clasifican en mayor proporción en el nivel alto y en menor proporción en el bajo que aquellos que no tienen dichos estudios.

Los conocimientos relativos a las bases curriculares y planes y programas de estudio presentan diferencias de acuerdo con el nivel de estudios. Los jefes técnicos con estudios de posgrado se clasifican en mayor proporción en alto nivel de conocimiento sobre documentos curriculares que aquellos que no tienen estudios de magíster. Adicionalmente, se observa que los de establecimientos particulares pagados tienen, en mayor proporción, un bajo nivel de conocimiento sobre los instrumentos curriculares respecto de sus pares de establecimientos municipales y particulares subvencionados.

Con relación a los conocimientos sobre evaluación, a nivel institucional y de aula se observan diferencias según nivel de estudios de los jefes técnicos: un 32\% de aquellos que tienen estudios de magíster se encuentra en el nivel alto de preparación, frente a un 20\% que no tiene ese nivel de estudios. 
Los conocimientos sobre evaluación de aprendizajes también presentan diferencias para los jefes técnicos con estudios de magíster y más años de experiencia en el cargo. Un 32\% de los jefes técnicos con estudios de magíster se encuentran en el nivel alto de preparación, frente a un $22 \%$ de jefes que no tiene ese nivel de estudios, y aquellos que tienen más años de experiencia en el cargo tienen una preparación más alta que aquellos con menos años, y viceversa.

Por otra parte, para los conocimientos sobre observación y retroalimentación de la práctica docente, se observa que los que tienen menos años de experiencia quedan mayormente clasificados en el nivel medio de conocimiento respecto de aquellos que tienen entre 7 y 14 años.

Al igual que en un caso anterior, llama la atención que los jefes técnicos de establecimientos con menores resultados Simce se declaren con mayor nivel de conocimiento de las políticas nacionales que aquellos de grupos con resultados de aprendizajes más altos.

Por último, en relación con las características de los jefes técnicos y sus establecimientos, y las diferencias respecto del nivel de preparación en conocimientos para el ejercicio del cargo, se observa que, en general, aquellos que tienen estudios de magíster presentan mayores niveles de preparación en cinco de nueve conocimientos consultados. Existen diferencias en algunos conocimientos con relación a los años de experiencia en el cargo del jefe técnico, la dependencia del establecimiento y los puntajes de resultados de aprendizajes (dos de nueve ítems). De esta manera, los estudios de magíster entregarían mayores conocimientos a los jefes técnicos para un ejercicio efectivo de su labor, de acuerdo con su percepción.

\section{Análisis comparado}

Los resultados muestran que los jefes técnicos se perciben con igual preparación para implementar las prácticas y conocimientos definidas en el MBDLE, observándose que solo un grupo pequeño, en promedio uno de cada cinco jefes técnicos, declara estar altamente preparado; en contraste, la misma cantidad se autopercibe con baja 
preparación. Al revisar los resultados de los jefes técnicos que se perciben altamente preparados en ambos componentes (prácticas y conocimientos), se observa que solo un $10 \%$ lo declara y, por otra parte, un $12 \%$ se percibe con baja preparación para implementar las prácticas y conocimientos descritas en el marco.

En relación con las dimensiones del marco, se obtiene que los jefes técnicos están preparados heterogéneamente para implementarlas, percibiéndose mejor preparados en unas por sobre otras y con cierta variabilidad al interior de ellas. En cuanto a los conocimientos, se perciben mejor preparados en aquellos asociados con la dimensión de enseñanza y aprendizaje que en los relativos a construir e implementar una visión estratégica compartida, que incluye aquellos saberes asociados a mejoramiento y cambio escolar, liderazgo y conocimiento de políticas nacionales, así como en la dimensión de "gestionando la convivencia", que considera conocimientos relativos a inclusión, equidad y estrategias para promoverlas, lo que posiblemente esté relacionado con el foco de su trabajo en los establecimientos, esto es, las materias técnicopedagógicas.

Coincidente con el componente de conocimiento, al analizar los resultados de las prácticas los jefes técnicos se sienten mejor preparados para implementar aquellas relacionadas con la dimensión de enseñanza y aprendizaje y, en menor medida, con la de desarrollo de capacidades profesionales de los equipos de establecimiento, lo que plantea un desafío importante para los equipos directivos, en el marco del desarrollo de capacidades que considera la ley de carrera docente.

Al comparar el grado de preparación de los jefes técnicos, de acuerdo con sus características, se obtiene que las relaciones más consistentes se refieren al nivel de estudios alcanzado en los conocimientos y los años de experiencia en el cargo para el componente de prácticas. Los jefes técnicos con estudios de magíster cuentan con una mejor preparación sobre algunos conocimientos definidos en el marco y aquellos con más años de experiencia en el cargo se perciben mejor preparados para implementar ciertas prácticas 
allí definidas. Así, un mayor nivel de preparación del jefe técnico, considerando la formación de posgrado y los años de experiencia en el cargo, se asocia con una mejor percepción de preparación para implementar algunas de las definiciones del marco.

Por último, y de acuerdo con los resultados sobre la preparación de los directores para ejercer un liderazgo efectivo (Rivero et al., 2019), se observa que los directores declaran contar con una mejor preparación que los jefes técnicos para implementar las prácticas del MBDLE ${ }^{6}$, con un 24\% en nivel alto de directores versus $21 \%$ de jefes técnicos; y los jefes técnicos se perciben mejor preparados en el componente de conocimientos, con un $17 \%$ en nivel alto de técnicos versus $12 \%$ de directores.

\section{Discusión y conclusión}

La investigación tendiente a estudiar la preparación de los líderes escolares entrega información valiosa, de modo de identificar las brechas para un ejercicio efectivo del cargo y proporcionar orientaciones con el fin de avanzar en su formación especializada. La literatura ha mostrado la centralidad de avanzar hacia el desarrollo de un liderazgo efectivo para mejorar los aprendizajes de los estudiantes y la calidad de la educación que estos reciben. A la fecha existen pocas investigaciones centradas en conocer la preparación de los directores de establecimientos, siendo aún más incipientes las relacionadas con conocer la de los encargados del área técnico-pedagógica para ejercer un liderazgo efectivo.

La escasa evidencia disponible muestra que los jefes técnicos dominan en mayor medida documentos de carácter administrativo (PME y estándares) y las bases curriculares; mientras que las áreas en que hay mayor carencia son la teoría del aprendizaje, la didáctica general y disciplinar, y el monitoreo curricular (Cortez y Zoro, 2016; Rodríguez, 2017; Ulloa y Gajardo 2017), además de conocimientos

6 La encuesta a directores fue aplicada a una muestra nacional de 575 ese año y de manera on line. En ambosestudiosse utilizóla misma metodologíaparala construcción delíndicesobre grado de preparación, variando algunas prácticas y conocimientos analizados de acuerdo con el ámbito de acción de cada miembro del equipo directivo. 
técnicos y estrategias para la resolución de conflictos, mejoramiento de las prácticas pedagógicas y competencias de coordinación y comunicación (Quiroga y Aravena, 2017; Beltrán, 2014). En relación con la preparación formal de los jefes técnicos para ejercer sus cargos, los estudios muestran una alta presencia de posgrados, divididos entre diplomados y magísteres, y un alto porcentaje de estos jefes que declara contar con formación relacionada con el cargo, concentrándose en las áreas de liderazgo y gestión, currículo y evaluación (MINEDUC, 2016; Rodríguez, 2017).

A partir de la opinión de los jefes técnicos de establecimientos de todas las dependencias administrativas de la Región Metropolitana, los resultados muestran que el MBDLE es un instrumento de política de liderazgo escolar conocido por los encargados del área técnicopedagógica. No obstante, si bien existe conocimiento de esta guía para el desarrollo de un liderazgo directivo efectivo, los resultados muestran que el grado de preparación autoreportado por los jefes técnicos para implementar las prácticas y los conocimientos es solo mediano. De esta manera, si bien el marco es conocido por los jefes técnicos, solo el 10\% de ellos se percibe altamente preparado respecto de las prácticas y conocimientos consultados, por lo que resulta ser un marco relativamente ambicioso y difícil de implementar, todo esto de acuerdo con el nivel de preparación declarado por los propios actores.

Al analizar los resultados según las dimensiones del marco, se obtiene que los jefes técnicos se perciben con mejor preparación respecto de la dimensión de enseñanza y aprendizaje y con menos en la de desarrollar las capacidades profesionales de sus equipos y de construir una visión estratégica compartida en materia de conocimientos, que incluye los conocimientos de políticas nacionales y los relativos a inclusión y equidad, lo que es consistente con el estudio del MINEDUC (2016). Esta menor preparación representa un desafío y alerta sobre eventuales dificultades para resguardar una implementación exitosa de políticas recientes, como la Política Nacional de Desarrollo Docente, que considera el desarrollo profesional al interior de las unidades escolares, y la ley de inclusión. En concreto, un grupo de jefes técnicos se percibe bajamente 
preparado en contenidos sobre políticas nacionales y normativa educacional, sobre inclusión y equidad, y sobre liderazgo y estrategias de mejora escolar. Adicionalmente, se percibe escasamente preparado para desarrollar prácticas relacionadas con promover y participar del desarrollo profesional de los docentes, así como con participar en sus procesos de selección.

Cabe mencionar que, si bien la preparación es informada por los mismos jefes técnicos, los resultados sugieren la presencia de brechas importantes de abordar para generar las capacidades que el MBDLE establece en los cargos de liderazgo y que, además, se requieren para una adecuada implementación de las políticas educacionales en curso en el sistema educativo chileno. Así, para que el marco se convierta en una guía que oriente efectivamente a los líderes escolares, esto es, para que sea factible y alcanzable, se presenta el desafío de generar espacios de formación especializados con este actor del equipo escolar, que ha sido, hasta ahora, poco visibilizado dentro de las políticas educacionales y de liderazgo escolar. Específicamente, para este cargo se observan debilidades en áreas que trascienden a la técnica pedagógica propiamente tal, las que, si bien pueden ser reforzadas a partir de los hallazgos mencionados, muestran mayores debilidades en las dimensiones de desarrollo profesional de los equipos de trabajos, así como en las habilidades de liderazgo, para construir una visión compartida y contribuir a una adecuada implementación de las políticas educacionales en curso.

Por otra parte, los resultados muestran que los jefes técnicos con estudios de posgrado - magíster en concreto- presentan asociaciones positivas consistentes con mayores grados de preparación para implementar las prácticas y conocimientos definidos en el marco. Esto indica que medidas que incentiven la especialización de los jefes técnicos pueden contribuir a la reducción de las brechas detectadas. Las áreas de formación necesarias de priorizar, de acuerdo con los resultados, serían habilidades para el desarrollo profesional del cuerpo docente y las competencias necesarias para asegurar una adecuada implementación de la normativa relacionada con la ley de inclusión. Por otra parte, los años de experiencia en el cargo también 
presentan relaciones positivas con un mayor grado de preparación en algunas prácticas y conocimientos, aspecto importante de considerar en las políticas o mecanismos de selección de este estratégico cargo dentro de las unidades escolares.

Finalmente, resulta conveniente avanzar, como se ha realizado en este caso con el cargo del jefe técnico, en explorar el nivel de preparación de otros miembros de los equipos directivos encargados de liderar las comunidades educativas, conocer cómo se complementan las habilidades y formación, e identificar las brechas de mejora necesarias de abordar con cada uno de ellos. De esta manera, es posible avanzar, sobre la base de evidencia empírica, una línea de formación de los cargos que componen los equipos directivos y conformar equipos cada vez más capaces de ejercer un liderazgo escolar efectivo de acuerdo con las definiciones de la guía del MBDLE, de modo de lograr una implementación exitosa de las políticas educacionales según las capacidades que éstas demandan de los actores claves de implementarlas, y contribuir al logro de mayores resultados de aprendizaje de los estudiantes con líderes escolares mejor preparados.

\section{Referencias}

Agencia de Calidad de la Educación. (2015). El liderazgo del equipo directivo en enseñanza básica y su relación con indicadores de desarrollo personal y social de suestablecimiento. Documento de trabajo para la comunidad escolar.

Barber, M. \& Mourshed, M. (2007). How the world's best-performing schools come out on top. London: McKinsey and Company.

Beltrán, J. (2014). Factores que dificultan la gestión pedagógica curricular de los jefes de unidades técnico pedagógicas. Revista Mexicana de Investigación Educativa, 19(62), 939-961.

Bellei, C., Valenzuela, J., Vanni, X. y Contreras, D. (2014). Lo aprendí en la escuela: ¿Cómo se logran procesos de mejoramiento escolar? Santiago, Chile: LOM.

Carbone, R. (2008). Situación del Liderazgo Educativo en Chile. MINEDUC y Universidad Alberto Hurtado, 1-30.

Cortez, M. y Zoro, B. (2016). El rol estratégico de los liderazgos medios al interior de las escuelas para la mejora escolar: una mirada desde la literatura internacional. Nota Técnica 8, Líderes Educativos. 
Day, C., Sammons, P., Hopkins, D., Harris, A., Leithwood, K., Gu, Q., Penlington, C., Mehta, P. \& Brown, E. (2009). The Impact of Leadership on Pupil Outcomes: Final Report to DSCF. Nottingham: Department of Children, Families \& Schools/National College of School Leadership.

De Nobile, J. (2018). Towards a theoretical model of middle leadership in schools. School Leadership \& Management, 395-416.

Flückiger, B., Lovett, S., Dempster, N. \& Brown, S. (2015). Middle Leaders: Career pathways and professional learning needs. Leading $\mathcal{E}$ Managing, 21(2), 60-74.

Hallinger, P. \& Heck, R. H. (1998). Exploring the principal's contribution to school effectiveness: 1980-1995. School effectiveness and school improvement, 9(2), 157-191. DOI: http://dx.doi. org/10.1080/0924345980090203

Leithwood, K., Day C., Sammons P., Harris A. \& Hopkins D. (2006). Successful school leadership: What it is and how it influences pupil learning. London: DfES and Nottingham: NCSL.

Marfán, J., González, R., Muñoz, G., Pascual, J., Valenzuela, P. y Weinstein,

J. (2012). Estudio comparado de liderazgo escolar: Aprendizajes para Chile a partir de los resultados PISA 2009. En: Evidencias para políticas públicas eneducación. ProyectoFONIDEN ${ }^{\circ}$ :FE11140. Santiago, Chile: Ministerio de Educación de Chile.

Marzano, R. J., Waters, T. \& McNulty, B. (2005). Schoolleadership that works: From research to results. Aurora, CO: ASCD and McREL.

Ministerio de Educación Chile. MINEDUC. (2015). Marco para la Buena dirección y el Liderazgo Escolar. Recuperado de: http://portales.mineduc. cl/ usuarios/cpeip/doc/201511131613560.MBD\&LE_2015.pdf

Ministerio de Educación Chile. MINEDUC. (2016). Estudio de caracterización de los equipos directivos escolares de establecimientos educacionales subvencionados urbanos de Chile. Santiago, Chile: MINEDUC.

Pont, B., Nusche, D. \& Moorman, H. (2009). Improving School Leadership. Policy and Practice, 1, OECD publishing.

Quiroga, M. \& Aravena, F. (2017). Jefes de UTP nóveles en Chile: desafíos del liderazgo pedagógico. Perspectiva Educacional, 76-97.

Rivero, R., Yáñez, T., Hurtado, C. y Strello, A. (2017). Estudio de opinión a líderes escolares y de niveles intermedios sobre políticas de liderazgo escolar. Recuperado de https://www.lidereseducativos.cl/wp-content/ uploads/2019/01/15.-Presentacion-Encuesta-Directivos.pdf 
Rivero, R., Yáñez, T. y Hurtado, C. (2019). Preparación para ejercer un liderazgo efectivo: estudio de opinión de directores escolares a partir del Marco de la Buena Dirección. Archivos Analíticos de Políticas Educativas, 27(117). DOI: https://doi.org/10.14507/epaa.27.4391 Este artículo forma parte del númeroespecial, Estándaresy Competencias en Gestión Educativa: Perspectivas Globales, Comparativas y Críticas, editado por Augusto Riveros and Wei Wei.

Robinson, V. M., Lloyd, C. A. \& Rowe, K. J. (2008). The impact of leadership on student outcomes: An analysis of the differential effects of leadership types. Educational administration quarterly, 44(5), 635-674. DOI: http:// dx.doi.org/10.1177/0013161X08321509

Rodríguez Molina, G. A. \& Gairín Sallán, J. (2017). Influence of the practices of pedagogical leadership in the educational pedagogical practices: case in Chile of the Pedagogical Technical Units. International Journal of Educational Leadership and Management, 6-29.

Silins, H. \& Mulford, B. (2002). Schools as learning organisations: The case for system, teacher and student learning. Journal of educational administration, 40(5), 425-446. DOI: http://dx.doi. org/10.1108/09578230210440285

Valenzuela, M. y Horn, A. (2012). Influencia del liderazgo directivo en los resultados de los estudiantes. En: J. Weinstein y G. Muñoz (eds), ¿Qué sabemos sobre los directores de escuela en Chile? (pp. 325-348). Santiago, Chile: Salesianos Impresores.

Volante, P. (2012). Liderazgo instruccional y logro académico en la educación secundaria en chile. En: J. Weinstein y G. Muñoz (eds), ¿Qué sabemos sobre los directores de escuela en Chile? (pp. 349-370). Santiago, Chile: Salesianos Impresores.

Weinstein, J. y Hernández, M. (2014). Políticas hacia el liderazgo directivo escolar en Chile: Una mirada comparada con otros sistemas escolares de América Latina. Psicoperspectivas, 13(3), 52-68.

Weinstein, J. y Muñoz, G. (2012). Introducción. En: J. Weinstein y G. Muñoz (eds), ¿Qué sabemos sobre los directores de escuela en Chile? (pp. 325- 348). Santiago, Chile: Salesianos Impresores. 